\title{
ON THE OPTIMAL EXERCISE BOUNDARY FOR AN AMERICAN PUT OPTION
}

\author{
GHADA ALOBAIDI AND ROLAND MALLIER
}

Received 18 July 2000 and in revised form 12 February 2001

An American put option is a derivative financial instrument that gives its holder the right but not the obligation to sell an underlying security at a pre-determined price. American options may be exercised at any time prior to expiry at the discretion of the holder, and the decision as to whether or not to exercise leads to a free boundary problem. In this paper, we examine the behavior of the free boundary close to expiry. Working directly with the underlying PDE, by using asymptotic expansions, we are able to deduce this behavior of the boundary in this limit.

\section{Introduction}

Options are derivative financial securities, giving the holder the right to sell (a put) or buy (a call) some underlying security at a specified price. They have many uses in the financial world, including hedging, speculating, generating income, and contributing to market completeness by expanding the universe of investment opportunities. Although options were in existence prior to 1973, when the Chicago Board Options Exchange (CBOE) introduced standardized, listed options, it was only after this date that the first secondary market for options came into existence and they became widely traded. Prior to the opening of the $\mathrm{CBOE}$, in practical terms, it was difficult if not impossible for the holder of an option to sell it, meaning that the holder either had to exercise the option or hold it till expiry.

Most options are either European, meaning that they can be exercised only at expiry, or American, meaning that they can be exercised at or before expiry. European options are comparatively easy to evaluate, with the wellknown Black-Scholes formula having been available to investors since 1973, the same year the $\mathrm{CBOE}$ opened its doors. American options are harder to

Copyright (c) 2001 Hindawi Publishing Corporation

Journal of Applied Mathematics 1:1 (2001) 39-45

2000 Mathematics Subject Classification: 91B28

URL: http://jam.hindawi.com/volume-1/S1110757X01000018.html 
evaluate. Because early exercise is possible, the holder is constantly faced with the decision as to whether to retain an option or exercise it, and that leads to a free boundary problem for the optimal exercise boundary which divides the region where exercise is optimal from the region where it is optimal to hold the option. To date, no closed form solution is known for this optimal exercise boundary, and instead we must rely on numerical solutions and approximations. One such approximation, which we employ here, is to study the optimal exercise boundary close to expiry, so that we can regard the time left till expiry as small and pose an expansion.

For the call, which gives the holder the right to buy the underlying stock, it is possible to use straightforward asymptotic analysis (Alobaidi [3] and Wilmott [8]) to find a series for the location of the optimal exercise boundary close to expiry. The motivation for doing this is that with relatively few terms, it is possible to obtain a fairly accurate answer extremely quickly, while a finite-difference scheme would take several minutes to arrive at the answer.

Turning to the optimal exercise boundary for the American put, two studies using alternate approaches are the motivation for the work presented here. Barles et al. [4] were able to find upper and lower bounds for the optimal exercise boundary near to expiration and then prove that these upper and lower bounds approached each other as expiration was approached, leading them to conclude that the value of the option behaved like

$$
S_{f}(t) \sim E\left(1-\sigma[(T-t)|\log (T-t)|]^{1 / 2}\right)
$$

for $t<T$ near $T$, where $E$ is the strike price of the option and $\sigma$ is the volatility. Kuske and Keller [6] derived an integral equation for the free boundary using a Green's function method and solved it iteratively. They stopped after three iterations, with the leading order term on their solution agreeing with that of Barles et al. [4]. In what follows, we will attempt to use an asymptotic analysis to analyse the free boundary near to expiration, and see why a naïve expansion will not work, and what must be done to make an expansion work. This allows us to recover the leading order result of the two studies mentioned above.

\section{Analysis}

It is well known that the value $P(S, t)$ of a put option on a dividend paying stock obeys the Black-Scholes PDE

$$
\frac{\partial P}{\partial t}+\frac{\sigma^{2} S^{2}}{2} \frac{\partial^{2} P}{\partial S^{2}}+\left(r-D_{0}\right) S \frac{\partial P}{\partial S}-r P=0,
$$

where $S$ is the price of the underlying, $r$ is the risk-free rate, $D_{0}$ is the (constant) dividend yield, and $\sigma$ is the volatility. The pay-off at expiry $(t=T)$ 
is given by $P(S, T)=\max (E-S, 0)$, subject to the boundary conditions that $\mathrm{P}(0, \mathrm{t})=\mathrm{E} e^{-\mathrm{r}(\mathrm{T}-\mathrm{t})}$ and $\mathrm{P}(\mathrm{S}, \mathrm{t}) \rightarrow 0$ as $\mathrm{S} \rightarrow \infty$. For an American put, the possibility of early exercise leads to the constraint $P(S, t) \geq \max (E-S, 0)$ for $t<$ $\mathrm{T}$, which is the pay-off that would be achieved by immediate exercise. One approach commonly taken with this system is to apply the transformation $\mathrm{S}=\mathrm{E} e^{\mathrm{x}}, \mathrm{t}=\mathrm{T}-2 \tau / \sigma^{2}, \mathrm{P}=\mathrm{E}-\mathrm{S}+\mathrm{E} p, \mathrm{k}_{1}=2 \mathrm{r} / \sigma^{2}$, and $\mathrm{k}_{2}=2\left(\mathrm{r}-\mathrm{D}_{0}\right) / \sigma^{2}$, which leads to the new equation:

$$
\frac{\partial p}{\partial \tau}=\frac{\partial^{2} p}{\partial x^{2}}+\left(k_{2}-1\right) \frac{\partial p}{\partial x}-k_{1} p+f(x)
$$

where the nonhomogeneous term $f(x)=\left(k_{1}-k_{2}\right) e^{x}-k_{1}$, subject to the payoff at expiry $p(x, 0)=\max \left(e^{x}-1,0\right)$ and the constraint $p(x, \tau) \geq \max \left(e^{x}-\right.$ $1,0)$ for $\tau>0$.

The first step in our analysis is to find the location of the free boundary at expiry. At $\tau=0$, if $x>0$, we have $p=0$ and $\partial p / \partial \tau=0$. This last derivative comes from evaluating the PDE (2.2) at $\tau=0$. However, if $x<0$ then at $\tau=0$, we have $p=0$ and $\partial p / \partial \tau=f(x)<0$. Because this derivative is negative, close to expiry, $p$ itself will also become negative, which violates the constraint $p(x, \tau) \geq \max \left(e^{x}-1,0\right)$, and so this tells us that the optimal exercise boundary starts at $x=0$ when $\tau=0$. This corresponds to $S=E$ in the original variables.

Having found the location of the free boundary at $\tau=0$, we now attempt to find the location of the boundary close to expiry, denoting this boundary by $x_{f}(\tau)$. In the original variables, we require (e.g., Wilmott [8]) that $P=0$ and $\partial P / \partial S=-1$ at $S=S_{f}(t)$, which corresponds to $p=\partial p / \partial x=0$ at $x=x_{f}(\tau)$. We know from above that $x_{f}(0)=0$, so we attempt to perform a local analysis in the vicinity of $x=0, \tau=0$, and we are interested in the region $x>x_{f}(\tau)$. To perform our analysis, we introduce the rescaled coordinates $x=v X, \tau=\mu \xi$, and $p(x, \tau)=\varepsilon \rho(X, \xi)$, where $\nu, \mu$, and $\varepsilon$ are small positive quantities. Substituting these new variables into our PDE and considering the possible balances between various terms leads us to conclude that $\mu=v^{2}=\varepsilon^{2}$, so that (2.2) becomes

$$
\begin{aligned}
\frac{\partial \rho}{\partial \xi}= & \frac{\partial^{2} \rho}{\partial X^{2}}+v\left[\left(k_{2}-1\right) \frac{\partial \rho}{\partial X}-k_{2}\right]+v^{2}\left[-k_{1} \rho+\left(k_{1}-k_{2}\right) X\right] \\
& +\left(k_{1}-k_{2}\right)\left(\frac{v^{3} X^{2}}{2 !}+\frac{v^{4} X^{3}}{3 !}+\frac{v^{5} X^{4}}{4 !}+\cdots\right),
\end{aligned}
$$

with the condition that $\rho(X, \xi=0) \sim X+v X^{2} / 2 !+v^{2} X^{3} / 3 !+v^{3} X^{4} / 4 !+\cdots$. To solve this, we attempt to expand $\rho$,

$$
\rho \sim \rho_{0}+v \rho_{1}+v^{2} \rho_{2}+\cdots .
$$


42 On the optimal exercise boundary for an American put option

Substituting this into (2.3), we get at leading order,

$$
\frac{\partial \rho_{0}}{\partial \xi}=\frac{\partial^{2} \rho_{0}}{\partial X^{2}}
$$

with the boundary condition that at $\xi=0, \rho_{0}(X, \xi=0)=X$. Writing $\rho_{0}=$ $\xi^{1 / 2} g_{0}(\eta)$, where the similarity variable $\eta=X \xi^{-1 / 2}$, we get an equation for go,

$$
g_{0}^{\prime \prime}+\frac{1}{2} \eta g_{0}^{\prime}-\frac{1}{2} g_{0}=0
$$

with a solution

$$
g_{0}(\eta)=\eta+C_{0}\left[2 e^{-\eta^{2} / 4}-\pi^{1 / 2} \eta \operatorname{erfc}\left(\frac{\eta}{2}\right)\right] .
$$

Taking the limit $\eta \rightarrow \infty$, we recover the limit $\xi=0$. Looking at the free boundary, from our rescaling we assume that we can write

$$
x_{f}(\tau)=x_{1} \tau^{1 / 2}+x_{2} \tau+x_{3} \tau^{3 / 2}+\cdots,
$$

so that the free boundary is located at

$$
X_{f}(\xi)=v^{-1} x_{f}(\tau)=x_{1} \xi^{1 / 2}+v x_{2} \xi+v^{2} x_{3} \xi^{3 / 2}+\cdots,
$$

and the boundary conditions that $p$ and $\partial p / \partial x$ vanish at the free boundary mean that, at leading order we require, at $X=x_{1} \xi^{1 / 2}$ we require $\rho_{0}=$ $\partial \rho_{0} / \partial X=0$, which translates into requiring that $g_{0}\left(x_{1}\right)=g_{0}^{\prime}\left(x_{1}\right)=0$. Applying this leads to the two equations

$$
\begin{gathered}
x_{1}+C_{0}\left[2 e^{-x_{1}^{2} / 4}-\pi^{1 / 2} x_{1} \operatorname{erfc}\left(\frac{x_{1}}{2}\right)\right]=0, \\
1-C_{0} \pi^{1 / 2} \operatorname{erfc}\left(\frac{x_{1}}{2}\right)=0,
\end{gathered}
$$

which means that we require $e^{-x_{1}^{2} / 4}=0$ or $x_{1}=\infty$, and this implies that our scaling is wrong.

Having seen our naïve expansion fail, the standard approach in a situation like this is to introduce logarithms (e.g., Goldstein [5] and Mallier [7]). This is because the derivative of $\log \tau$ is $\tau^{-1}$, and this provides missing terms in the expansion. We therefore assume that near to expiration the free boundary behaves like

$$
x_{\mathrm{f}}(\tau)=x_{1} \tau^{1 / 2}(\log \tau)^{\gamma_{0}}+\cdots
$$

for some power $\gamma_{0}$ of the logarithm, rather than the simple expansion (2.8) as we had assumed earlier. Our expansion for the option value therefore takes 
the form

$$
\begin{aligned}
p(x, \tau) \sim & \lambda^{\gamma_{0}}\left[g_{0}^{(0)}(\eta)+\lambda^{-1} g_{1}^{(0)}(\eta)+\lambda^{-2} g_{2}^{(0)}(\eta)+\cdots\right] \\
& +\tau^{1 / 2} \lambda^{\gamma_{1}}\left[g_{0}^{(1)}(\eta)+\lambda^{-1} g_{1}^{(1)}(\eta)+\lambda^{-2} g_{2}^{(1)}(\eta)+\cdots\right]+\cdots
\end{aligned}
$$

where $\eta=x \tau^{-1 / 2}$ and $\lambda=\log \tau$, with the free boundary given by $x_{f}(\tau)=$ $\tau^{1 / 2} Y_{f}(\tau)$, where

$$
\begin{aligned}
Y_{f}(\tau) \sim & \lambda^{\delta_{0}}\left[y_{0}^{(0)}+\lambda^{-1} y_{1}^{(0)}+\lambda^{-2} y_{2}^{(0)}+\cdots\right] \\
& +\tau^{1 / 2} \lambda^{\delta_{1}}\left[y_{0}^{(1)}+\lambda^{-1} y_{1}^{(1)}+\lambda^{-2} y_{2}^{(1)}+\cdots\right]+\cdots
\end{aligned}
$$

Substituting the expansion (2.13) into the governing equation (2.2), we find that the leading order $\tau^{0} \lambda^{\gamma}$ term obeys

$$
g_{0}^{(0)^{\prime \prime}}(\eta)+\frac{1}{2} \eta g_{0}^{(0)^{\prime}}(\eta)=0,
$$

which has a solution which vanishes as $\tau \rightarrow 0$,

$$
g_{0}^{(0)}(\eta)=C_{0}^{(0)} \operatorname{erfc}\left(\frac{\eta}{2}\right)
$$

Amongst the $\tau^{1 / 2}$ terms, there will be a homogeneous term at $\mathcal{O}\left(\tau^{1 / 2} \lambda^{0}\right)$ which satisfies

$$
g_{0}^{(1)^{\prime \prime}}+\frac{1}{2} \eta g_{0}^{(1)^{\prime}}-\frac{1}{2} g_{0}^{(1)}=0
$$

which has a solution that satisfies the condition as $\tau \rightarrow 0$,

$$
g_{0}^{(1)}(\eta)=\eta+C_{0}^{(1)}\left[2 e^{-\eta^{2} / 4}-\pi^{1 / 2} \eta \operatorname{erfc}\left(\frac{\eta}{2}\right)\right] .
$$

We need this to balance the leading order contribution from $g_{0}^{(0)}$ (and subsequent terms such as $\left.g_{1}^{(0)}, g_{2}^{(0)}, \ldots\right)$ at the free boundary. To evaluate $g_{0}^{(0)}$ at the free boundary, we recall that

$$
\operatorname{erfc}\left(\frac{\eta}{2}\right) \sim \frac{2 e^{-\eta^{2} / 4}}{\eta \pi^{1 / 2}}\left(1-\frac{2}{\eta^{2}}+\frac{12}{\eta^{4}}+\cdots\right) .
$$

Crucial in our analysis is the behavior of the term $e^{-\eta^{2} / 4}$ at the free boundary, $\eta=Y_{f}$,

$$
\begin{aligned}
e^{-\gamma_{f}^{2} / 4}= & \exp \left[-\frac{\lambda^{2 \gamma_{0}} y_{1}^{(0) 2}}{4}\right] \\
& \times \exp \left[-\frac{\lambda^{2 \gamma_{0}}}{2 \lambda}\left(y_{0}^{(0)} y_{1}^{(0)}+\lambda^{-1}\left(y_{0}^{(0)} y_{2}^{(0)}+\frac{y_{1}^{(0) 2}}{2}\right)+\mathcal{O}\left(\lambda^{-2}\right)\right)+\mathcal{O}\left(\tau^{1 / 2}\right)\right] .
\end{aligned}
$$


To help us with the conditions at the free boundary, we need the leading order contribution from $g_{0}^{(1)}$ to balance that from $g_{0}^{(0)}$. To do this, we require the term $\exp \left[-\lambda^{2 \gamma_{0}} y_{1}^{(0) 2} / 4\right]$ to be of the form $\exp [(1 / 2) \log \tau]=\tau^{1 / 2}$ (because $g_{0}^{(1)}$ is multiplied by $\tau^{1 / 2}$ in the expansion). Recalling that $\lambda=\log \tau$, this can only hold if $\delta_{0}=1 / 2$ and $y_{0}^{(0) 2}=-2$, so that $\exp \left[-\lambda^{2} \gamma_{0} y_{1}^{(0) 2} / 4\right]=$ $\tau^{1 / 2}$. Physically, we also require that $x_{f}(\tau)$ be a monotone decreasing function of $\tau$, so that the free boundary slopes downwards. Thus we have recovered the leading order behavior of the free boundary close to expiry,

$$
x_{f}(\tau) \sim-(-2 \tau \log \tau)^{1 / 2}+\mathcal{O}\left(\tau^{1 / 2}(\log \tau)^{-1 / 2}, \tau\right) .
$$

This result agrees with earlier studies using different methods. Kuske and Keller [6] used a Green's function to convert the problem into an integral equation, which they then solved iteratively, stopping after three iterations. Their result was

$$
\begin{aligned}
S_{f} & \sim E\left(1-\left[2 \sigma^{2}(T-t) \log \left(\frac{\sigma}{r[2 \pi(T-t)]^{1 / 2}}\right)\right]^{1 / 2}\right) \\
& \sim E\left[1-\sigma \sqrt{T-t}\left(\sqrt{|\log (T-t)|}+\frac{\log (\sigma /(3 r \sqrt{2 \pi}))}{\sqrt{|\log (T-t)|}}+\cdots\right)\right] .
\end{aligned}
$$

Converting their solution to the variables used in this study, we recover

$$
x \sim \tau^{1 / 2}\left(-\sqrt{-2 \log \tau}-\log \left(\frac{\sigma^{2}}{6 r \sqrt{\pi}}\right) \sqrt{-\frac{2}{\log \tau}}+\cdots\right)+\cdots,
$$

the leading order term of which agrees with our result.

\section{Discussion}

In Section 2, we considered the optimal exercise boundary of an American put option, and used asymptotic analysis to find the behavior of that boundary close to expiry, finding that at leading order $x_{f}(\tau) \sim-(-2 \tau \log \tau)^{1 / 2}$. This agrees with previous studies by Barles et al. [4] and Kuske and Keller [6], who used different methods to consider the same problem.

In a sense, this result, although expected, is disappointing because it suggests that asymptotic analysis is much less practical for a put than for a call. It should be recalled that a call option allows the holder to buy the underlying stock, while a put gives the holder the right to sell it. For a call (Alobaidi [3] and Wilmott [8]), a straightforward series of the form $x_{f} \sim$ $x_{0}+x_{1} \tau^{1 / 2}+x_{2} \tau+\cdots$ is possible for the optimal exercise boundary, meaning that a very good approximation for the location of the boundary close to expiry can be obtained with fairly few terms, and such an approximation could be evaluated extremely quickly numerically, whereas a standard finitedifference solution might well take several minutes. 
Mathematically, the difference between the put and the call in terms of the asymptotic analysis presented here is the appearance of the logarithmic terms in the series for the put. It follows that for the put, because of the presence of these $\log \tau$ terms, it is not possible to get the same level of accuracy as for the call, even if a large number of terms is calculated. Further, it appears that the calculation of subsequent terms would be extremely challenging, as it would appear that terms of the form $\log (\log \tau), \log (\log (\log \tau)), \ldots$, will enter the series at later orders. A preliminary investigation of that aspect of the problem is given in Alobaidi [3].

Finally, we mention in passing that in this study, as was the case with those of Barles et al. [4] and Kuske and Keller [6], we have assumed that the underlying stock prices follow a log-normal random walk, or geometric Brownian motion, and that the value of an option obeys the Black-Scholes equation. Ait-Sahalia (Ait-Sahalia [1] and Ait-Sahalia and Lo [2]) has considered the case where the price of the underlying stock obeys a more complex stochastic process; he showed that the price of an option could be found using state-price densities or SPD's, which can be estimated non-parametrically using market data, rather than relying on specific assumptions. The BlackScholes model can be recovered from Ait-Sahalia's approach as a special case.

\section{References}

[1] Y. Ait-Sahalia, Nonparametric pricing of interest rate derivative securities, Econometrica 64 (1996), 527-560.

[2] Y. Ait-Sahalia and A. W. Lo, Nonparametric estimation of state-price densities implicit in financial asset prices, J. Finance 53 (1998), 499-547.

[3] G. Alobiadi, American options and their strategies, Ph.D. thesis, University of Western Ontario, London, Canada, 2000.

[4] G. Barles, J. Burdeau, M. Romano, and N. Samsoen, Critical stock price near expiration, Math. Finance 5 (1995), no. 2, 77-95. Zbl 866.90029.

[5] S. Goldstein, On laminar boundary-layer flow near a point of separation, Proceedings of the Cambridge Philosophical Society 26 (1930), 1-18.

[6] R. E. Kuske and J. B. Keller, Optimal exercise boundary for an American put option, Applied Mathematical Finance 5 (1998), 107-116.

[7] R. Mallier, On the parametric model of western boundary outflow, Stud. Appl. Math. 91 (1994), no. 1, 17-25. MR 95a:76020. Zbl 796.76024.

[8] P. Wilmott, Derivatives, the Theory and Practice of Financial Engineering, Wiley, Chichester, 1998.

Ghada Alobaidi: Department of Applied Mathematics, The University of Western Ontario, London, Canada ON N6A 5B7

E-mail address: alobaidi@math.uregina.ca

Roland Mallier: Department of Applied Mathematics, The University of Western 0ntario, London, Canada 0N N6A 5B7

E-mail address: mallier@uwo.ca 


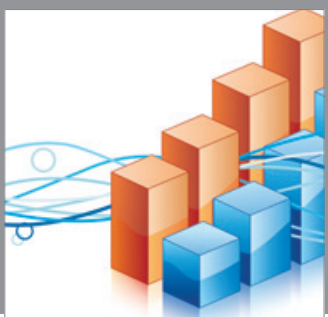

Advances in

Operations Research

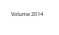

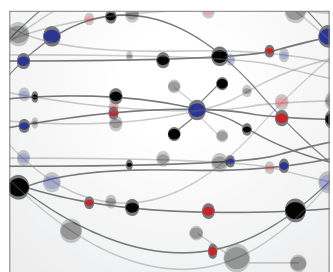

\section{The Scientific} World Journal
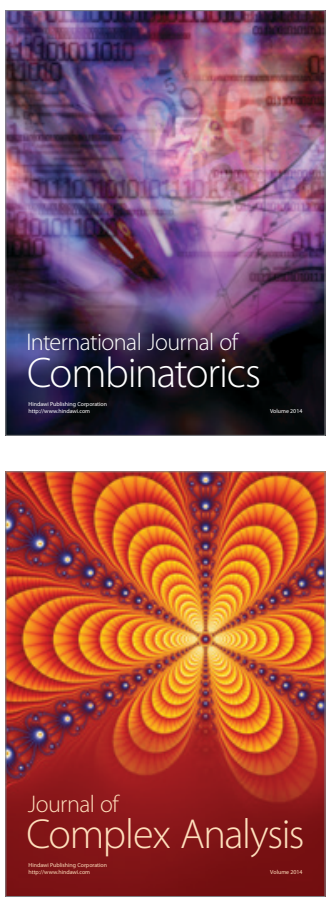

International Journal of

Mathematics and

Mathematical

Sciences
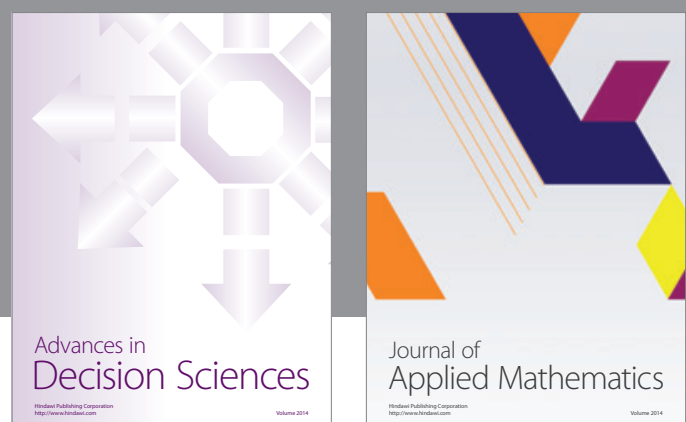

Journal of

Applied Mathematics
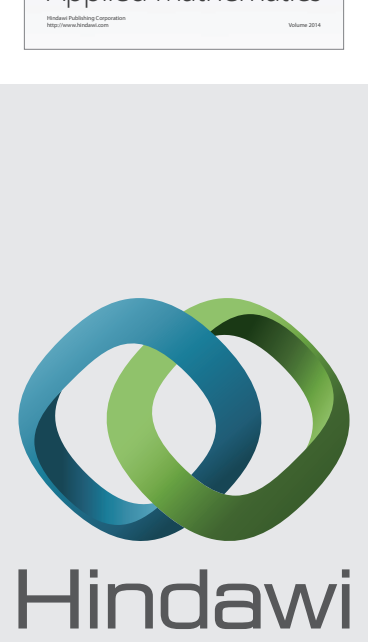

Submit your manuscripts at http://www.hindawi.com
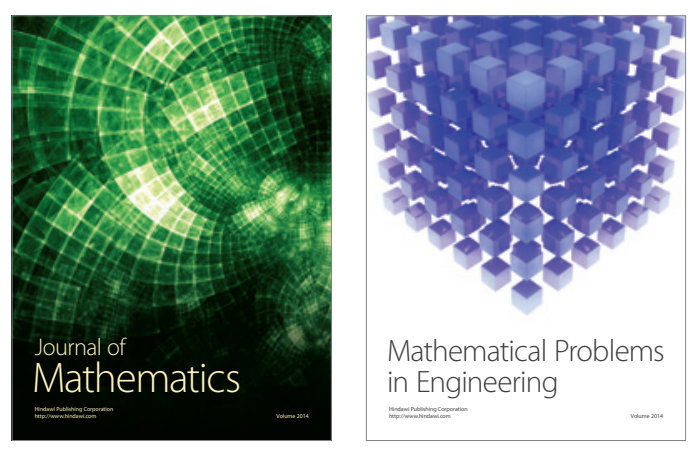

Mathematical Problems in Engineering
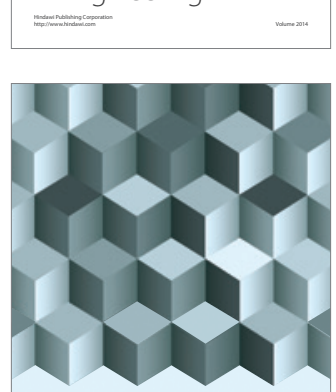

Journal of

Function Spaces
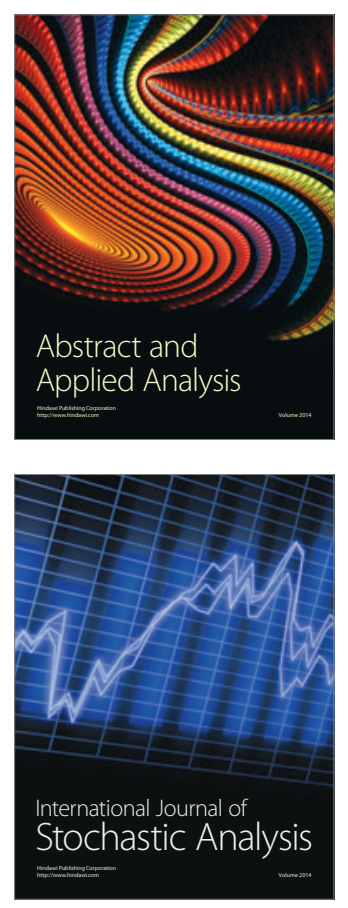

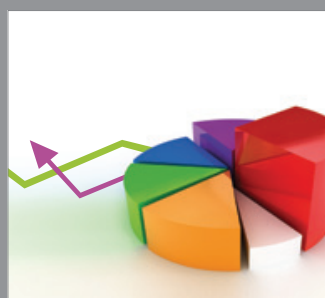

ournal of

Probability and Statistics

Promensencen
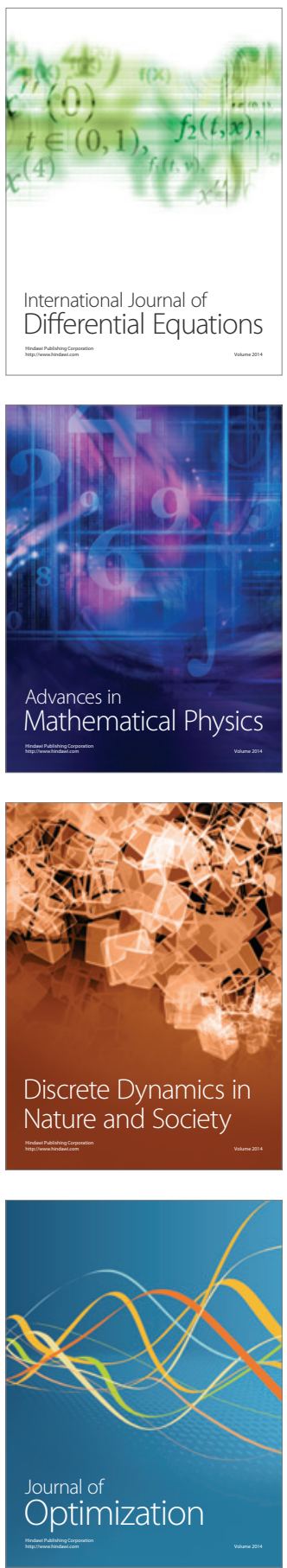